\title{
Quantum transport within a background medium: Fluctuations versus Correlations
}

\author{
Holger Fehske, Andreas Alvermann, and Gerhard Wellein
}

\begin{abstract}
We investigate transport within some background medium by means of an effective lattice model with a novel form of fermion-boson coupling. The bosons correspond to local fluctuations of the background. The model captures the principal transport mechanisms that apply to a great variety of physical systems, and can be applied, e.g. in the one-particle sector, to describe the motion of lattice and spin polarons, or the dynamics of a particle coupled to a bath. Performing large-scale numerical simulations on the HLRB-II at LRZ Munich, based on highly efficient variational Lanczos and Chebyshev moment expansion techniques, we analyse the newly proposed model by exactly calculating the single quasiparticle effective mass, groundstate dispersion and spectral function, as well as the Drude weight and the optical conductivity for an infinite one-dimensional system. Moreover, for the half-filled band case, we establish a metal-insulator quantum phase transition by analysing the particle-particle/boson correlations and photoemission spectra.
\end{abstract}

\section{Motivation}

The motion of a particle that interacts strongly with some background medium is a constantly recurring theme in condensed matter physics. Media which commonly occur are ordered spin backgrounds as in the $t$ - $J$ model of doped Mott insulators [1, 2] or vibrating lattices, as in the Holstein, Peierls or

Holger Fehske and Andreas Alvermann

Ernst-Moritz-Arndt-Universität Greifswald, Institut für Physik, Felix-Hausdorff-Str. 6, D-17489 Greifswald

Gerhard Wellein

Regionales Rechenzentrum Erlangen, Martensstraße 1, D-91058 Erlangen 
quantised Su-Schrieffer-Heeger models for polarons or charge density waves (CDW) $[3,4,5]$.

As the particle moves it creates local distortions of substantial energy in the medium, e.g. local spin or lattice fluctuations, which may be able to relax. Their relaxation rate determines how fast the particle can move. In this sense particle motion is not free at all; the particle is continuously creating a string of distortions but can move on "freely" at a speed which gives the distortions time to decay.

This picture is very general with wide applicability, e.g. to charge transport in high- $T_{c}$ superconductors, colossal-magnetoresistive materials, MX chains, mesoscopic devices like quantum wires, and presumably even biological systems $[6,7,8]$.

To be more specific let us consider a hole in a two-dimensional (2D) antiferromagnet, a situation characteristic for the quasi-2D cuprate superconductors (cf. Fig. 1). In a classical Néel background motion of the hole creates a string of misaligned spins. This string effect strongly restricts propagation [9]. If however spins exchange quantum-mechanically distortions of the spin background can "heal out" by local spin fluctuations with a rate controlled by the antiferromagnetic exchange parameter $J$. This way the hole can move coherently but with a reduced bandwidth proportional to $J[10]$.

In this contribution we study a spinless model which nevertheless captures some of this two-fold physics in the single charge carrier limit and also exhibits a quantum phase transition from a metallic to an insulting phase at halffilling $[14,15]$.

\section{Theoretical model}

Let us consider the Hamiltonian

$$
H=-t_{b} \sum_{\langle i, j\rangle} c_{j}^{\dagger} c_{i}\left(b_{i}^{\dagger}+b_{j}\right)-\lambda \sum_{i}\left(b_{i}^{\dagger}+b_{i}\right)+\omega_{0} \sum_{i} b_{i}^{\dagger} b_{i}+\frac{N \lambda^{2}}{\omega_{0}}
$$

for fermionic particles $(c)$ coupled to bosonic fluctuations $(b)$ of frequency $\omega_{0}$. The third term gives the energy of the bosons; the constant energy shift $N \lambda^{2} / \omega_{0}$ guarantees finite energy for $N \rightarrow \infty$.

In (1) a fermion, e.g. an electron, emits or absorbs a local boson every time it hops between nearest neighbour lattice sites $i$ and $j$, but hopping creates (destroys) a bosonic fluctuation in the background medium only on the site the particle leaves (enters). The four fundamental hopping processes that constitute the kinetic energy in the $\lambda=0$ Hamiltonian are 


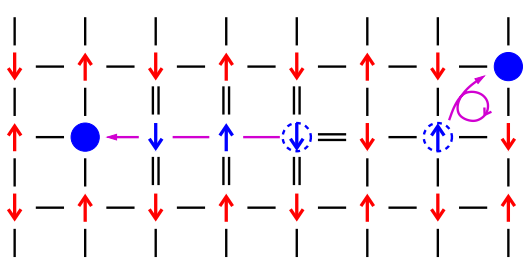

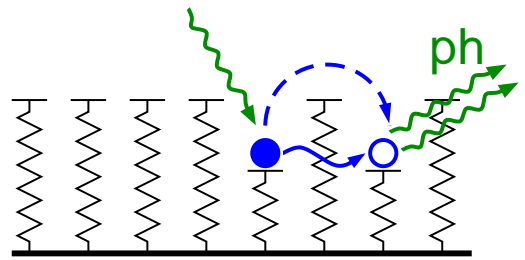

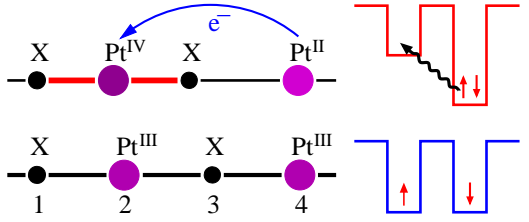

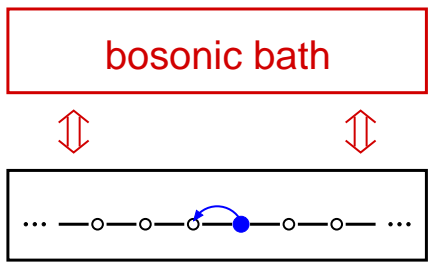

Fig. 1 Quantum transport in correlated/fluctuating background media. Upper left panel: Charge transport in a 2D antiferromagnet. A hole creates frustrated antiferromagnetic bonds if it hops. On the other hand, if the hole travels around a square one and a half times, it unwinds the string and finds itself translated to a next-nearest-neighbour site with the background spins undisturbed [11]. Upper right panel: Charge transport in MX chains [5]. Schematic view of the mixed-valence ground state and intervalence charge transfer to an excited state without charge disproportionation. In the process of lattice relaxation a charge-transfer exciton is formed. Lower left panel: Small polaron transport [12]. Here are two mechanisms at play: Itinerant (coherent) polaron motion is possible on an extremely (exponentially) reduced energy scale, where all phonon numbers remain the same during the hop (diagonal transition). On the other hand the number of phonons might be changed (non-diagonal transition) and each hop may be considered as a statistically independent event. Clearly the particle loses its phase coherence by these phonon emission or absorption (inelastic scattering) processes. Lower right panel: Charge transport in a quantum wire coupled to a bosonic (heat) bath [13]. Note that in all cases transport is strongly boson affected or even controlled - as a result two transport channels may evolve.

$$
\begin{aligned}
& R_{i}=c_{i+1}^{\dagger} c_{i} b_{i}^{\dagger}|\cdot \stackrel{\curvearrowright}{\odot} \cdot\rangle \mapsto|\cdot \star \star \odot\rangle \\
& L_{i}=c_{i-1}^{\dagger} c_{i} b_{i}^{\dagger}|\cdot \stackrel{\curvearrowleft}{\odot} \cdot\rangle \mapsto|\odot \star \cdot\rangle
\end{aligned}
$$

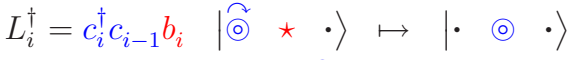

$$
\begin{aligned}
& \left.R_{i}^{\dagger}=c_{i}^{\dagger} c_{i+1} b_{i} \mid \cdot * \text { ๑) }\right\rangle \mapsto|\cdot \odot \cdot\rangle \text {, }
\end{aligned}
$$

where $\odot$ stands for the fermion, $\star$ for a boson, · for an unoccupied lattice site, and circular arrows mark the hop of the fermion under the application of the operators.

The $\lambda$ term allows a boson to decay spontaneously, thereby avoiding the string effect (compare $\lambda$ with $J$ ). Thus $t$ - $J$-like quasiparticle transport becomes possible. In the "classical limit" $\lambda \rightarrow 0$ coherent quasiparticle motion is suppressed as in the $t-J_{z}$ (Ising spin) model. However, even at $\lambda=0$, when transport is fully boson-assisted, there exist processes that propagate 
the particle but restore the boson vacuum. The lowest-order process of this kind comprises 6 steps,

$$
\begin{aligned}
& |\widetilde{\odot} \cdot \cdot\rangle \rightarrow|\star \tilde{\odot} \cdot\rangle \rightarrow|\star \star \star \tilde{\odot}\rangle \rightarrow|\star \stackrel{\hat{\rho}}{\star} \star\rangle \rightarrow|\tilde{\odot} \star \star\rangle \rightarrow|\cdot \tilde{\odot} \star\rangle \rightarrow|\cdot \cdot \odot\rangle \\
& \left|\begin{array}{cc}
\uparrow & \downarrow \\
\odot & \uparrow
\end{array}\right\rangle \rightarrow\left|\begin{array}{cc}
\odot & \downarrow \\
\uparrow & \uparrow
\end{array}\right\rangle \rightarrow\left|\begin{array}{cc}
\downarrow & \odot \\
\uparrow & \uparrow
\end{array}\right| \rightarrow\left|\begin{array}{ll}
\downarrow & \uparrow \\
\uparrow & \odot
\end{array}\right| \rightarrow\left|\begin{array}{ll}
\downarrow & \uparrow \\
\odot & \uparrow
\end{array}\right| \rightarrow\left|\begin{array}{ll}
\odot & \uparrow \\
\downarrow & \uparrow
\end{array}\right\rangle \rightarrow\left|\begin{array}{ll}
\uparrow & \odot \\
\downarrow & \uparrow
\end{array}\right\rangle,
\end{aligned}
$$

where in steps $1-3$, three bosons are excited, which are consumed in steps 4-6 (see upper row). Thus " $R_{i}^{(6)}=L_{i+2}^{\dagger} L_{i+1}^{\dagger} R_{i}^{\dagger} L_{i+2} R_{i+1} R_{i}$ " acts as " $c_{i+2}^{\dagger} c_{i}$ ", i.e. is a one-dimensional representation of the "Trugman path" [11] in a Néelordered spin background (lower row). In this way the highly correlated manyparticle vacuum of the spin model is translated to the bosonic vacuum.

In order to "visualise" the coherent hopping channel hidden in (1), we perform a simple unitary transformation $b_{i} \mapsto b_{i}+t_{f} / 2 t_{b}$ of $H$ :

$$
H^{\prime}=-t_{f} \sum_{\langle i, j\rangle} c_{j}^{\dagger} c_{i}-t_{b} \sum_{\langle i, j\rangle} c_{j}^{\dagger} c_{i}\left(b_{i}^{\dagger}+b_{j}\right)+\omega_{0} \sum_{i} b_{i}^{\dagger} b_{i} .
$$

Obviously $H^{\prime}$ describes two transport channels, one of unrestricted hopping $\propto t_{f}=2 \lambda t_{b} / \omega_{0}$, and a second of boson-controlled hopping $\propto t_{b}$. While for $t_{b}=0$ the model reduces to that of a free particle, for $t_{b} \neq 0$ the physics of the model is governed by two ratios: The relative strength $t_{b} / t_{f}$ of the two transport mechanisms, and the rate of bosonic fluctuations $t_{b} / \omega_{0}$. Therein the model also resembles common electron-phonon models like the Holstein or SSH model. Note, however, that the limit $\omega_{0} \rightarrow 0$ does not immediately lead to a semi-classical description established for these electron-phonon models since the electron does not couple exclusively to oscillator coordinates $\propto$ $\left(b_{i}+b_{i}^{\dagger}\right)$.

In order to solve the model (1) or (2) we combine the exact diagonalisation (ED) and kernel polynomial methods briefly outlined in Sec. 4. For a detailed description of these highly accurate and efficient numerical techniques we refer to very recent reviews $[16,17,18]$. Let us emphasise that the variational Lanczos results presented below for the one-particle sector are numerically exact for an infinite system.

\section{Results and discussion}

\subsection{Transport in the one-particle sector}

For a quantitative analysis of transport properties we employ the regular part of the optical conductivity 


$$
\sigma_{r e g}(\omega)=\sum_{n>0} \frac{\left|\left\langle\psi_{n}|j| \psi_{0}\right\rangle\right|^{2}}{\omega_{n}} \delta\left(\omega-\omega_{n}\right)
$$

Here $\left|\psi_{n}\right\rangle$ label the eigenstates of the one-fermion system with excitation energy $\omega_{n}=E_{n}-E_{0}$, and $\left|\psi_{0}\right\rangle$ is the ground state. The current operator is given by

$$
\begin{aligned}
j & =j_{f}+j_{b} \\
& =\mathrm{i} t_{f} \sum_{i} c_{i+1}^{\dagger} c_{i}-c_{i}^{\dagger} c_{i+1}+\mathrm{i} t_{b} \sum_{i} c_{i+1}^{\dagger} c_{i} b_{i}^{\dagger}-c_{i}^{\dagger} c_{i+1} b_{i}-c_{i-1}^{\dagger} c_{i} b_{i}^{\dagger}+c_{i}^{\dagger} c_{i-1} b_{i} .
\end{aligned}
$$

Generally the Drude weight $D$ serves as a measure of the coherent, free particle like transport, and fulfils the f-sum rule

$$
-D=\frac{E_{k i n}}{2}+\int_{0}^{\infty} \sigma_{r e g}(\omega) d \omega
$$

where $E_{k i n}=\left\langle\psi_{0}\left|H-\omega_{0} \sum_{i} b_{i}^{\dagger} b_{i}\right| \psi_{0}\right\rangle$ is the kinetic energy.

For a free particle, the Drude weight is given by $D=t_{f}$, and $-D / E_{k i n}=$ 0.5 , while $-D / E_{k i n} \ll 0.5$ for diffusive transport in the presence of strong fluctuations. We can therefore characterise different transport regimes through the ratio $-D / E_{k i n}$ (see Fig. 2). The curve for large boson frequency, $\omega_{0} / t_{b}=$ 2.0 , shows that in a wide range of $t_{f} / t_{b}$ transport is quasi-free with $-D / E_{k i n} \lesssim$ 0.5 . For smaller $\omega_{0} / t_{b}$, as the number of fluctuations is larger, $-D / E_{k i n}$ is decreased due to scattering. The smaller $\omega_{0} / t_{b}$, the slower $-D / E_{k i n}$ tends to its asymptotic value 0.5 for $t_{f} / t_{b} \rightarrow \infty$. This shows how the crossover from a coherent regime with quasi-free transport to an incoherent regime with diffu-

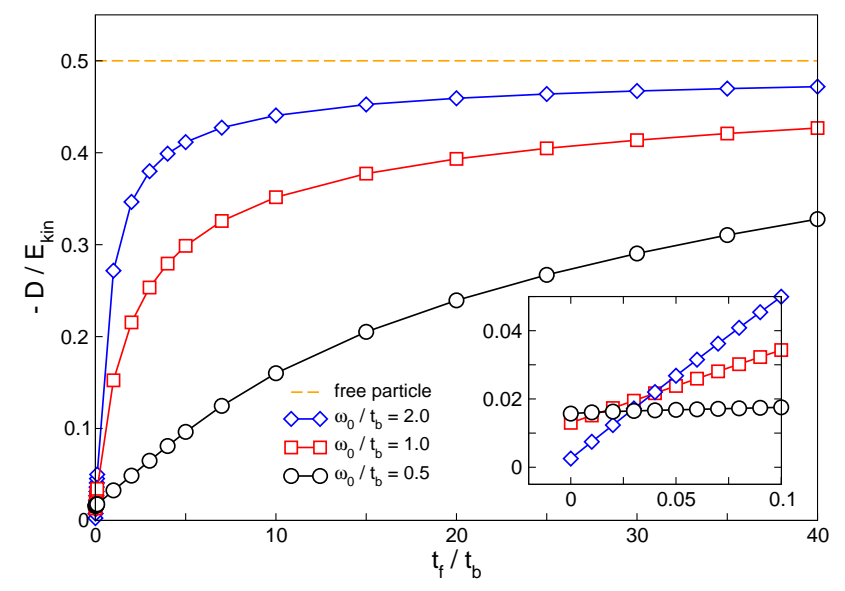

Fig. 2 Drude weight $D$ scaled to the kinetic energy $E_{k i n}$, where the inset displays the region $t_{f} / t_{b} \ll 1$ magnified. Note that $D=1 /\left(2 m^{*}\right)$. 
sive transport is controlled by $t_{b} / \omega_{0}$. For small $t_{f} / t_{b}$, when boson-controlled hopping is the dominating transport process, $D$ increases with decreasing $\omega_{0}$ (see inset). This is the regime of boson-assisted hopping, where transport is mediated by vacuum-restoring (6-step) processes (cf. the discussion in the previous section). We note that $D$ at $t_{f}=0$ saturates as $\omega_{0} \rightarrow 0$ [15].

By Kohn's formula, $D=1 /\left(2 m^{*}\right)$, the Drude weight is related to the effective mass $m^{*}$ which becomes renormalised if $t_{b}>0$. The effective mass can be obtained independently from the ground-state dispersion $E(k)$ via $1 / m^{*}=\left.\frac{\partial^{2} E}{\partial k^{2}}\right|_{k=0}(E(k)$ denotes the lowest energy value in each $k$-sector $)$. Using these data for $D$ in (5), it is worth mentioning that the f-sum rule is satisfied numerically to at least six digits in the whole parameter regime of our model (1).

To complete the characterisation of the system we determined the particleboson and particle-particle correlations in the ground state $\left|\psi_{0}\right\rangle$ :

$$
\begin{aligned}
& \chi_{e b}(i-j)=\frac{1}{N_{e}} \sum_{i}\left\langle\psi_{0}\left|c_{i}^{\dagger} c_{i} b_{i+j}^{\dagger} b_{i+j}\right| \psi_{0}\right\rangle, \\
& \chi_{e e}(i-j)=\frac{1}{N_{e}^{2}} \sum_{i}\left\langle\psi_{0}\left|c_{i}^{\dagger} c_{i} c_{i+j}^{\dagger} c_{i+j}\right| \psi_{0}\right\rangle .
\end{aligned}
$$

Moreover we calculated the spectral density of single-particle excitations associated with the injection of an electron with wave-vector $k, A^{+}(k, \omega)$ (inverse photoemission), and the corresponding quantity for the emission of an electron, $A^{-}(k, \omega)$ (photoemission),

$$
A^{ \pm}(k, \omega)=\sum_{n}\left|\left\langle\psi_{n}^{ \pm}\left|c_{k}^{ \pm}\right| \psi_{0}\right\rangle\right|^{2} \delta\left[\omega \mp \omega_{n}^{ \pm}\right],
$$

where $c_{k}^{+}=c_{k}^{\dagger}, c_{k}^{-}=c_{k}$, and $\left|\psi_{0}\right\rangle$ is the ground state in the $N_{e}$-particle sector while $\left|\psi_{n}^{ \pm}\right\rangle$denote the $n$-th excited states in the $N_{e} \pm 1$-particle sectors with $\omega_{n}^{ \pm}=E_{n}^{ \pm}-E_{0}$.

Figure 3 gives a survey of our numerical results for the one-particle sector. Let us first discuss the regime of small-to-intermediate boson frequencies $\omega_{0} / t_{b}$ and hopping ratios $t_{b} / t_{f}$ (see left column). The particle-boson correlation function (uppermost panel) shows that bosonic fluctuations are rather weakly correlated. Of course, they form a cloud surrounding the particle, but are not further correlated. This resembles the situation for a large Holstein (lattice) polaron, where the role of bosonic fluctuations is taken by optical phonons $[19,20]$. The spectral function $A^{+}(k, \omega)$ supports this picture. The spectral weight is distributed along the "free" dispersion $-2 t_{f} \cos k$, like for a weakly bound particle-boson excitation. Around $k= \pm \pi$ the over-damped character of particle motion is very prominent. Comparing with $E(k)$, we see that the quasiparticle weight is negligible away from $k=0$, and a welldefined quasiparticle band does not exist. For $k= \pm \pi / 2$ almost all weight resides in a single coherent peak at $\omega=0$. A particle injected with $k= \pm \pi / 2$ 

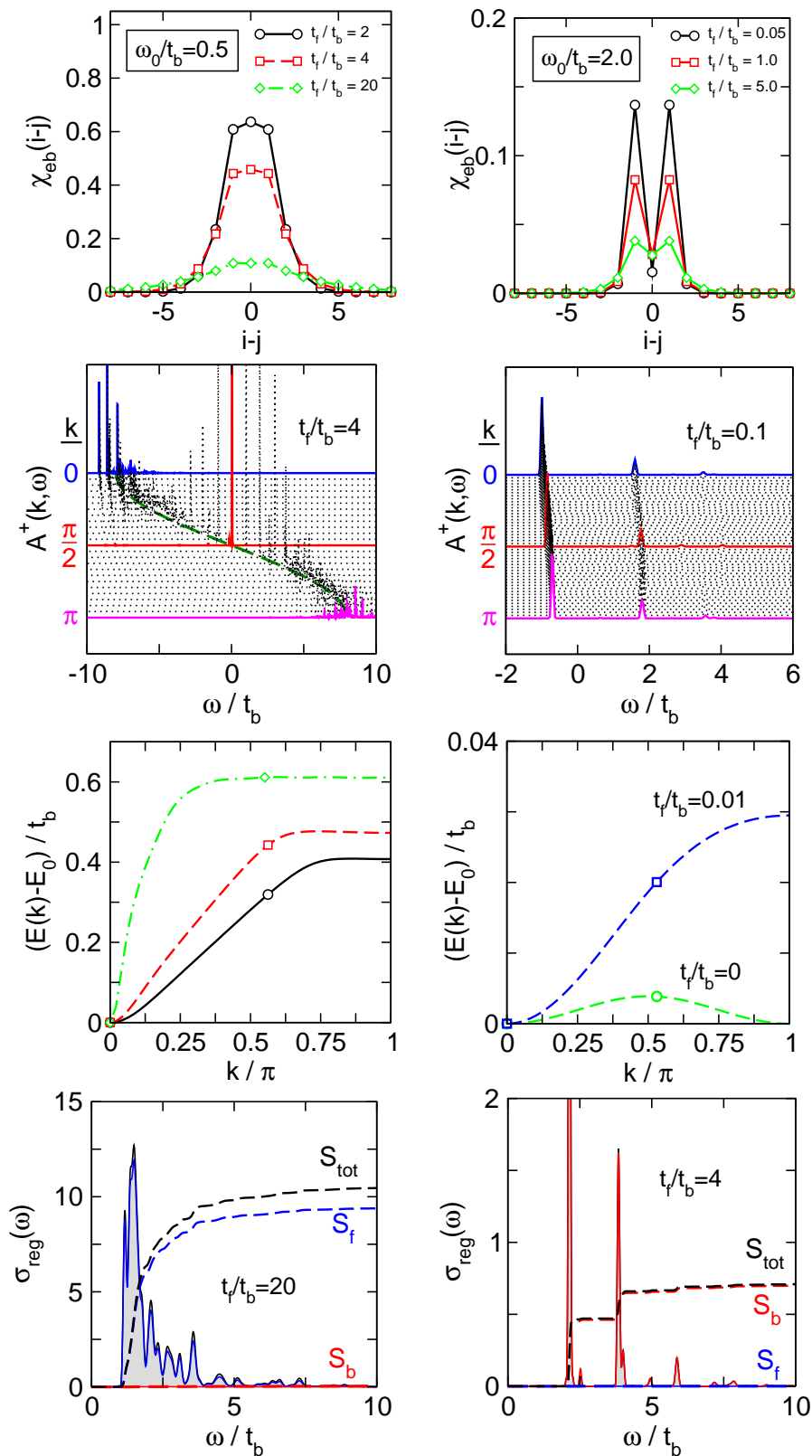

Fig. 3 Electron-boson correlations $\chi_{e b}(i-j)$, spectral function $A^{+}(k, \omega)$, band dispersion $E(k)$, and optical response $\sigma_{r e g}(\omega)$ (from top to bottom) in the single-particle transport model (2). The left (right) column gives data for $\omega_{0} / t_{b}=0.5\left(\omega_{0} / t_{b}=2.0\right)$, i.e. in the fluctuation (correlation) dominated regime. 
therefore propagates almost unaffected by bosonic fluctuations. In a sense, the system is transparent at this energy, similar to e.g. a potential (double) well at certain resonant energies. The regular part of the optical conductivity is dominated by a broad incoherent absorption continuum above a phonon absorption threshold (see bottom panel). This again is evocative of a large Holstein polaron situation [20]. To analyse the relative importance of the two transport processes $j_{f}$ and $j_{b}$, we have shown the corresponding contributions $\sigma_{f}(\omega), \sigma_{b}(\omega)$ to $\sigma_{r e g}(\omega)$ separately (note that generally $\left.\sigma_{r e g}(\omega) \neq \sigma_{f}(\omega)+\sigma_{b}(\omega)\right)$. $S_{t o t}$ (and similar $S_{f}, S_{b}$ ) gives the integrated conductivity $S_{\text {tot }}(\omega)=\int_{0}^{\omega} \sigma_{r e g}\left(\omega^{\prime}\right) d \omega^{\prime}$.

From this regime, characterised by rather diffusive transport, we can evolve in two directions. First, if we increase $t_{b} / t_{f}$, the contribution of bosoncontrolled hopping to the conductivity begins to dominate [15]. If we further increase $t_{b} / t_{f}$ while keeping $t_{b} / \omega_{0}$ large, strong but uncorrelated bosonic fluctuations develop. As a result, the spectral function $A^{+}(k, \omega)$ will become fully incoherent, and $-D / E_{\text {kin }}$ is small. Apparently, the large number of bosonic fluctuations prevents strong correlations.

In the second direction, for large $t_{b} / t_{f}$ and rather small $t_{b} / \omega_{0}$, the number of fluctuations is reduced (cf. Fig. 3, right column). Then strong correlations evolve, e.g. in $\chi_{e b}(i-j)$ (see uppermost panel). Now the single-particle spectral function $A^{+}(k, \omega)$ consists of a few, well separated peaks (bands). This indicates that the model shows collective particle-boson dynamics, i.e. a dressed quasiparticle exists, like a spin/magnetic polaron in the $t$ - $J$ model $[10,21]$. As a particular feature of the correlated transport mechanism which dominates for $t_{b} / t_{f} \gg 1$, the quasiparticle dispersion $E(k)$ develops a $k \rightarrow k+\pi$ symmetry for $t_{b} / t_{f} \rightarrow \infty$. At $t_{f}=0$ the model therefore shows an electronic topological transition, for which the hole doped $t-J$ model provides a specific example. The optical conductivity is now entirely given by the contribution from boson-controlled hopping, but does not show the absorption continuum we found for diffusive transport (note that, although $-D / E_{k i n}$ must be small for large $t_{b} / t_{f}$, it is much larger than for large $\left.t_{b} / \omega_{0}\right)$.

The correlated transport mechanism for $t_{b} / t_{f} \gg 1$ is best understood in the limit $t_{f}=\lambda=0$. Then, the particle can only move by creating bosonic fluctuations, i.e. transport is fully boson-assisted. By the six step process discussed in Sect. 2 and similar higher order processes the particle is itinerant even at $t_{f}=0$, with a finite, though small, Drude weight. Since in any hop the boson number changes by one, any vacuum-restoring process propagates the particle by an even number of sites. This immediately explains, why $E(k)$ for $t_{f}=0$ has period $\pi$. The weight of the six step process scales as $t_{b}^{6} / \omega_{0}^{5}$. Thus boson-assisted transport dominates for large $\left(t_{b} / \omega_{0}\right)^{5}\left(t_{b} / t_{f}\right)$. In this regime, the mobility of the particle increases if $\omega_{0}$ decreases, as vacuum-restoring processes become energetically more favourable. This explains the opposite dependence of $D$ on $\omega_{0}$ apparent in the inset of Fig. 2 for $t_{f} / t_{b} \ll 1$ and $t_{f} / t_{b} \gtrsim 1$. 


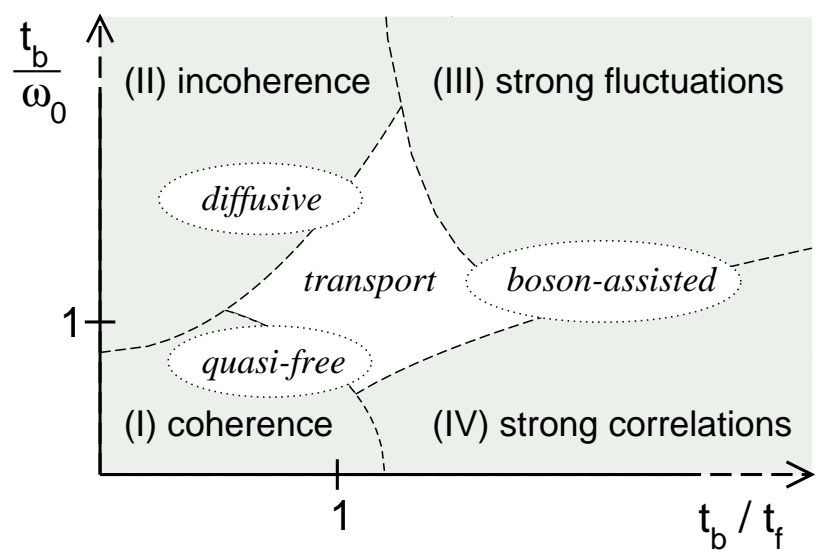

Fig. 4 Schematic view of the four physical regimes described by Hamiltonian (2).

Altogether Fig. 4 schematically displays the physics contained in our new model (1). In the limit of small $t_{b} / t_{f}$ (left side), transport takes place through unrestricted hopping. There, the model essentially describes motion of a particle coupled to a bosonic bath, when any bosonic fluctuations reduce the mobility of the particle. For small $t_{b} / \omega_{0}$ (regime I), the number of bosons is small. The particle propagates almost coherently, and transport resembles that of a free particle. If $t_{b} / \omega_{0}$ is larger (regime II), the number of bosons increases, and the bosonic timescale is slower than that of unrestricted hopping. Therefore bosonic fluctuations mainly act as random, incoherent scatters, and the particle loses its coherence. The transport is then diffusive, with a short mean free path. In the second limiting case, for large $t_{b} / t_{f}$ (right side), transport takes mainly place through boson-controlled hopping, i.e. particle motion relies on the existence of bosons, which are created and consumed in the hopping process. For large $t_{b} / \omega_{0}$ (regime III), transport is limited by strong scattering off uncorrelated bosonic fluctuations (similar to regime II). For small $t_{b} / \omega_{0}$ however (regime IV), the bosons instantly follow the particle motion and strong correlations develop, leading to collective particle-boson dynamics. In this way boson-controlled hopping acts in two opposing ways: Depending on how many correlations between the bosons persist, it may either limit transport as a result of scattering off random fluctuations (regime $\mathrm{I}+\mathrm{II}$ ), but may also enhance transport through correlated emission and absorption of bosons (regime IV). To conclude, in the single-particle sector, the Hamiltonian (1) provides a reduced but realistic description of fundamental aspects of transport in the presence of bosonic fluctuations. 


\subsection{Metal-insulator transition for the half-filled band case}

It is well-known that many quasi one-dimensional materials like MX-chains, ferroelectric perovskites, conjugated polymers, or organic charge transfer salts are very susceptible to structural distortions driven by the electron-phonon interaction [22]. Probably the most famous example is the Peierls instability [23] of 1D metals: As the temperature is lowered the system creates a periodic variation in the carrier density, a CDW, by shifting the ions from their symmetric positions. For the half-filled band case such a static dimerisation of the lattice opens a gap at the Fermi surface; as a result the metal gives way to a (Peierls) insulator. Related Holstein-type models are capable to describe this metal-insulator transition scenario: At $T=0$ they exhibit a spontaneous broken-symmetry CDW ground state, above a (finite) critical electron-phonon coupling strength (if finite-frequency phonons are taken into account) $[24,25,26]$. Then naturally the question arises, whether our bosoncontrolled hopping model (1) shows also a quantum phase transition from a metallic to an insulating phase at certain commensurate band fillings. Clearly the free hopping channel $\left(\propto t_{f}\right)$ is expected to act against any correlation induced CDW, but also strong bosonic fluctuations, i.e. large $t_{b} / \omega_{0}$, will tend to destroy long-range charge order.

To address this problem we have performed a large-scale ED study of the Hamiltonian (1) on the HLRB-II at the LRZ Munich. Unfortunately, the very efficient variational Hilbert space construction employed for the oneparticle sector in the preceding section cannot easily be extended to finite electron density. Thus, the computational requirements of the ED studies are determined by the total dimension $D_{t o t}=\left(\begin{array}{c}N \\ N\end{array}\right)\left(\begin{array}{c}N^{+}+N_{b} \\ N_{b}\end{array}\right)$ of the Hilbert space, where $N$ is the number of lattice sites, $N_{e}$ the number of electrons, and $N_{b}$ the maximum number of bosons retained. A typical production job for the complete photoemission spectrum for a system with $N=12, N_{e}=N / 2$, and $N_{b}=15$ at a given parameter set $t_{f} / t_{b}$ and $\omega_{0} / t_{b}$ runs on 300 processor cores of HLRB-II for more than 100 hours of wallclocktime, i.e. consumes more than 30000 CPU hours. To check for convergence concerning $N$ and $N_{b}$ we have computed selected parameters with $N=16\left(N_{b}=9\right)$ and $M=17(N=12)$ (maximum matrix dimensions about $D_{t o t}=4.8 \times 10^{10}$ ) requiring runs on up to 1000 processor cores. In principle, we could extend our studies to much larger matrix dimensions $\left(D_{\text {tot }}>40 \times 10^{10}\right)$ on HLRB-II but load balancing problems at high CPU counts prevented a reasonable use of HLRB-II, as described in more detail in Sect. 5 .

Figure 5 displays both the inverse $\left[A^{+}(k, \omega)\right]$ and direct $\left[A^{-}(k, \omega)\right]$ photoemission spectra for the $k= \pm \pi / 2$ sector where the gap is expected to appear at half-filling. At $t_{f} / t_{b}=1$ and $\omega_{0}=2\left(t_{b}=1\right.$ sets the energy scale hereafter), we found no energy gap between the photoemission and inverse photoemission signals. Consequently charge excitations are gapless at the 


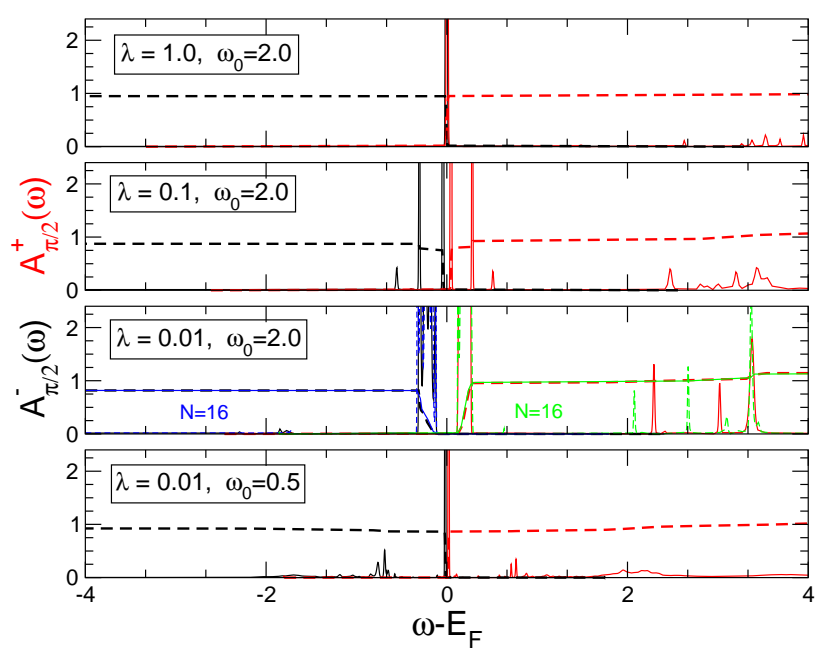

Fig. 5 Inverse photoemission spectra $A_{k}^{+}(\omega)$ (red) and photoemission spectra $A_{k}^{-}(\omega)$ (black) for the model (1) at half-filling $\left(N_{e} / N=0.5\right)$. Shown are results at the Fermi wavenumber $k_{F}= \pm \pi / 2$ and different $\lambda=\omega_{0} t_{f} /\left(2 t_{b}\right), \omega_{0}$, based on a finite-cluster diagonalisation with $N=12$ sites, $N_{b}=15$ bosons, and periodic boundary conditions. Green (blue) curves are the corresponding data for $N=16$ with $N_{b}=9$ bosons. All energies are measured in units of $t_{b}=1$.

Fermi momentum $k_{F}$ (Fermi energy $E_{F}$ ). Again the system is completely transparent at $k=\pi / 2$ (cf. the jump of the integrated spectral weight), but now, different from the one-particle case, this is the Fermi momentum, i.e. the model describes a perfect metal. As the calculation of the whole spectral function shows, most of the spectral weight of $A^{-}(k, \omega)\left[A^{+}(k, \omega)\right]$ resides in the uppermost [lowest] peaks in each $k$-sector [27]. This tendency is enhanced if we increase $t_{f} / t_{b}(\lambda)$ or $\omega_{0}$, whereby a quasi-free quasiparticle dispersion develops.

If we decrease $t_{f} / t_{b}(\lambda)$ keeping $\omega_{0}=2$ fixed a gap opens at about $\lambda=0.1$ in the photoemission spectra. The gap increases as $\lambda$ gets smaller (cf. middle panels of Fig. 5). Since the Fermi energy lies within the gap the system typifies an insulator. Of course, dealing with finite systems only, we are not in the position to draw any definite conclusion concerning the existence of a finite critical $\lambda_{c}$ in the thermodynamic limit. $\lambda_{c}$ may scale to zero as $N \rightarrow \infty$. However, comparing the $N=12$ and $N=16$ data, the finite-size dependence of the gap is found to be almost negligible, which gives some indication that $\lambda_{c}>0$ might be possible.

The transition to a CDW is driven by boson-controlled hopping $\propto t_{b}$. In the CDW state, where e.g. the even (odd) sites of the lattice are occupied (unoccupied), hopping of a fermion to a neighbouring site is possible. Since we then gain kinetic energy of the order $t_{b}^{2} / \omega_{0}$ per fermion, the CDW state 
is the ground state at large $\omega_{0}$ (and small $\lambda$ ), when excitation of bosons is suppressed. This mechanism resembles the Hubbard model at large $U$, when double occupancy is suppressed, and kinetic energy favours the antiferromagnetic state. Note the difference to the Peierls transition in the Holstein model, where the CDW is accompanied by a strong lattice deformation involving many phonons. Hence the Peierls CDW occurs favourably at small phonon frequencies [25].

That the observed metal-insulator transition is indeed driven by evolving correlations in the background medium - as e.g. for the Mott-Hubbard insulator - is corroborated by the weakening and finally closing of the excitation gap if the boson energy $\omega_{0}$ is reduced at fixed $\lambda$ (see lowest panel of Fig. 5). In this way the ability of the background to relax is enhanced, fluctuations overcome correlations and the system turns back to a metallic state. At the same time the spectral weight is transferred from the coherent to the incoherent part of the photoemission spectra, especially for $k$ away from from $k_{F}$ where the lineshape is dominated by rather broad bosonic signatures [27].

The CDW structure of the insulating state becomes apparent by investigating the particle-particle $\left[\chi_{e e}(i-j)\right]$ and particle-boson $\left[\chi_{e b}(i-j)\right]$ correlation functions (see Fig. 6). The even-odd modulation of the charge density away from a singled out site $i$ of the first particle is clearly visible. We note that the charge structure factor $S_{c}(\pi)$ increases by a factor of about two in going from $\lambda=0.1$ to $\lambda=0.01$ for the 16 -site system with $\omega_{0}=2$ [27].

In the CDW, where the even sites are occupied, every hop of a fermion excites a boson at an even site. This gives a large contribution to $\chi_{e b}(i-j)$ at neighbouring sites $|i-j|=1$, and at even sites $|i-j|=2,4,6, \ldots$ Since the CDW involves only few bosons, this contribution is the dominant contribution in first order of $t_{b} / \omega_{0}$. This explains why the boson density is large at sites with large fermion density, although the hopping term $t_{b}$ creates bosons at neighbouring sites of a fermion.

\section{Numerical approach}

\subsection{Ground-state properties}

Despite the great simplification brought by generic models, such as the Hamiltonian (1) studied in this contribution, theoretical investigations remain difficult whenever a quantum many-particle problem has to be solved with high accuracy to determine correlation effects on physical properties beyond the mean-field level. Therefore, in order to analyse the ground state, spectral and thermodynamic properties of these models, theorists have turned to numerical simulations. Among the various approaches, ED is presently one of the best controlled numerical methods because it allows an approximation-free 


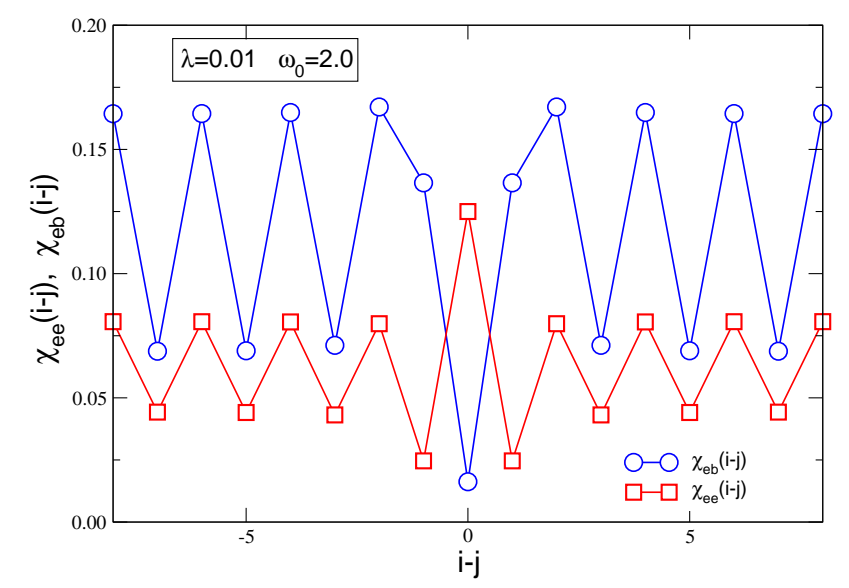

Fig. 6 Particle-particle and particle-boson correlations in the ground state of (1) with $\lambda=0.01$ and $\omega_{0}=2.0\left(N=16, N_{b}=9\right)$.

treatment of coupled electron-phonon models in the whole parameter range. As a precondition we have to work in general with finite systems and apply a well-defined truncation procedure for the phonon sector [28].

At least for the single-electron Holstein model,

$$
H=-t \sum_{\langle i, j\rangle} c_{i}^{\dagger} c_{j}+\omega_{0} \sum_{i} b_{i}^{\dagger} b_{i}-g \omega_{0} \sum_{i} c_{i}^{\dagger} c_{i}\left(b_{i}^{\dagger}+b_{i}\right),
$$

a variational basis can be constructed in such a way that the ground-state properties of the model can be computed numerically exact in the thermodynamic limit $[29,20]$. This technique can easily be adapted to the electronboson Hamiltonian (1). The approach is based on a clever way of constructing the electron-boson Hilbert space which can systematically be expanded in order to achieve high accuracy results with rather modest computational resources. The variational space is built up starting from an initial state, e.g. the electron at the origin without any boson, and acting repeatedly ( $\mathrm{L}$ times) with the off-diagonal hopping $t_{f}$ and $t_{b}$ terms of the Hamiltonian (1). A basis state is added if it is connected by a non-zero $t_{f}$ - or $t_{b}$-matrix element to a state previously generated, i.e., states in generation L are obtained by acting L times with the off-diagonal terms. Only one copy of each state is retained. Importantly, all translations of these states on an infinite lattice are included. According to Bloch's theorem $a_{j}=\mathrm{e}^{\mathrm{i} k j} a_{0}$, where $a_{j}$ is a set of complex amplitudes related to the states in the "unit cell" $j$. For each momentum $k$ the resulting numerical problem is then to diagonalise a Hermitian matrix of dimension of about $(\mathrm{D}+1)^{\mathrm{L}}$ (with $\mathrm{D}$ being the spatial dimension). Note that the error in the ground-state energy decreases exponentially with L. Thus in most cases $10^{4}-10^{6}$ basis states are sufficient to obtain an 8-10 digit accuracy 
for $E_{0}$. The ground-state energy calculated this way is variational for the infinite system.

To determine the eigenvalues of large sparse Hermitian matrices $H^{D}$, iterative (Krylov) subspace methods like Lanczos [30] and variants of Davidson [31] diagonalisation techniques are frequently applied [17]. Starting out from an arbitrary (random) initial state $\left|\varphi_{0}\right\rangle$, having finite overlap with the true ground state $\left|\psi_{0}\right\rangle$, the Lanczos algorithm recursively generates a set of orthogonal states (Krylov vectors):

$$
\left|\varphi_{l+1}\right\rangle=H^{D}\left|\varphi_{l}\right\rangle-a_{l}\left|\varphi_{l}\right\rangle-b_{l}^{2}\left|\varphi_{l-1}\right\rangle,
$$

where $a_{l}=\left\langle\varphi_{l}\left|H^{D}\right| \varphi_{l}\right\rangle /\left\langle\varphi_{l} \mid \varphi_{l}\right\rangle, b_{l}^{2}=\left\langle\varphi_{l} \mid \varphi_{l}\right\rangle /\left\langle\varphi_{l-1} \mid \varphi_{l-1}\right\rangle, b_{0}^{2}=0$, and $\left|\varphi_{-1}\right\rangle=0$. Obviously, the representation matrix $\left[T^{L}\right]_{l, l^{\prime}}=\left\langle\varphi_{l}\left|H^{D}\right| \varphi_{l^{\prime}}\right\rangle$ of $H^{D}$ is tridiagonal in the $L$-dimensional Hilbert space spanned by the $\left\{\left|\varphi_{l}\right\rangle\right\}_{l=0, \ldots, L-1}$, where $L \ll D$. Applying the Lanczos recursion (10), the eigenvalues $E_{n}$ and eigenvectors $\left|\psi_{n}\right\rangle$ of $H^{D}$ are approximated by $E_{n}^{L}$ and $\left|\psi_{n}^{L}\right\rangle=\sum_{l=0}^{L-1} c_{n, l}^{L}\left|\varphi_{l}\right\rangle$, respectively, where the L coefficients $c_{n, l}^{L}$ are the components of the $n$-th eigenvector of $T^{L}$ with eigenvalue $E_{n}^{L}$. The eigenvalue spectrum of $T^{L}$ can easily be determined using standard routines from libraries such as EISPACK. Increasing $L$ we check for the convergence of an eigenvalue of $T^{L}$ in a specific energy range. So we can avoid spurious eigenvalues for fixed Lanczos dimension $L$ which disappear as one varies $L$ [30].

Note that the convergence of the Lanczos algorithm is excellent at the edges of the spectrum (the ground state for example is obtained with high precision using at most $\sim 200$ Lanczos iterations) but rapidly worsens inside the spectrum. So Lanczos is suitably used only to obtain the ground state and a few low lying excited states.

\subsection{Spectral properties}

The numerical calculation of spectral functions,

$$
\begin{aligned}
A^{O}(\omega) & =-\lim _{\eta \rightarrow 0^{+}} \frac{1}{\pi} \operatorname{Im}\left[\left\langle\psi_{0}\left|O^{\dagger} \frac{1}{\omega-H+E_{0}+i \eta} O\right| \psi_{0}\right\rangle\right] \\
& =\sum_{n=0}^{D-1}\left|\left\langle\psi_{n}|O| \psi_{0}\right\rangle\right|^{2} \delta\left[\omega-\left(E_{n}-E_{0}\right)\right]
\end{aligned}
$$

where $O$ is the matrix representation of a certain operator $\hat{O}$ (e.g., the creation operator of an electron with wavenumber $k$ if one wants to calculate the single-particle spectral function, or the current operator if one is interested in the optical conductivity), involves the resolvent of the Hamilton matrix $H$. The idea behind the kernel polynomial method (KPM) [16, 17] 
is to expand $A^{O}(\omega)$ in a finite series of $(M+1)$ Chebyshev polynomials $T_{m}(x)=\cos [m \arccos (x)]$. Since the Chebyshev polynomials are defined on the real interval $[-1,1]$, we apply first a simple linear transformation to the Hamiltonian and all energy scales: $X=(H-b) / a, x=(\omega-b) / a$, $a=\left(E_{\max }-E_{\min }\right) /[2(1-\epsilon)]$, and $b=\left(E_{\max }+E_{\min }\right) / 2$ (the small constant $\epsilon$ is introduced in order to avoid convergence problems at the endpoints of the interval - a typical choice is $\epsilon \sim 0.01$ which has only $1 \%$ impact on the energy resolution [32]). Then the expansion reads

$$
A^{O}(x)=\frac{1}{\pi \sqrt{1-x^{2}}}\left(\mu_{0}^{O}+2 \sum_{m=1}^{M} \mu_{m}^{O} T_{m}(x)\right)
$$

with the coefficients (moments)

$$
\mu_{m}^{O}=\int_{-1}^{1} d x T_{m}(x) A^{O}(x)=\left\langle\psi_{0}\left|O^{\dagger} T_{m}(X) O\right| \psi_{0}\right\rangle .
$$

Eq. (12) converges to the correct function for $M \rightarrow \infty$. The moments

$$
\mu_{2 m}^{O}=2\left\langle\phi_{m} \mid \phi_{m}\right\rangle-\mu_{0}^{O} \quad \text { and } \quad \mu_{2 m+1}^{O}=2\left\langle\phi_{m+1} \mid \phi_{m}\right\rangle-\mu_{1}^{O}
$$

can be efficiently obtained by repeated parallelised matrix vector multiplication (MVM) [17], where $\left|\phi_{m+1}\right\rangle=2 X\left|\phi_{m}\right\rangle-\left|\phi_{m-1}\right\rangle$ but now $\left|\phi_{1}\right\rangle=X\left|\phi_{0}\right\rangle$ and $\left|\phi_{0}\right\rangle=O\left|\psi_{0}\right\rangle$, with $\left|\psi_{0}\right\rangle$ determined by Lanczos diagonalisation.

As is well known from Fourier expansion the series (12) with $M$ finite suffers from rapid oscillations (Gibbs phenomenon) leading to a poor approximation to $A^{O}(\omega)$. To improve the approximation the moments $\mu_{m}$ are modified $\mu_{m} \rightarrow g_{m} \mu_{m}$, where the damping factors $g_{m}$ are chosen to give the "best" approximation for a given $M$. This modification is equivalent to a convolution of the infinite series with a smooth approximation $K_{M}(x, y)$ to $\delta(x-y)$, a so-called approximation kernel. The appropriate choice of this kernel, that is of $g_{m}$, e.g. to guarantee positivity of $A^{O}(\omega)$, lies at the heart of KPM. We mainly use the Jackson kernel which results in a uniform approximation whose resolution increases as $1 / M$. In view of the uniform convergence of the expansion, KPM is a method tailored to the calculation of spectral properties. Most importantly, spectral functions obtained via KPM are not subject to uncontrolled or biased approximations: The accuracy of its outcome does only depend on the expansion depth $M$, and can be made as good as required by just increasing $M$. Of course one is restricted to finite systems of moderate size whose associated Hamilton matrix does not exceed available computational resources. 


\section{Load balancing and scalability issues on HLRB-II}

The physical parameter space accessible to ED studies of interacting quantum systems such as the Hamiltonian (1) is mainly determined by the size of the sparse matrix involved in the MVM step of the numerical approaches presented in Sect. 4. Even for the small lattice sizes considered in Sect. 3.2 the matrix dimensions easily exceed ten billion and a parallel, memory saving implementation of the sparse MVM step becomes indispensable. Choosing a "out-of-core" implementation, where even the non-zero entries of the matrix are not stored but recomputed in each MVM step, limits the memory requirements to those essential to the numerical approach, e.g. the Lanczos vectors, and some auxiliary fields for buffering messages.

The direct product formulation of the Hilbert space $\mathcal{H}=\mathcal{H}_{f} \otimes \mathcal{H}_{b}$ for interacting fermion-boson Hamiltonians offers a straightforward parallelisation approach and allows for a memory efficient implementation of the "out-ofcore" MVM step. Using the complete basis set $\{|s\rangle=|f\rangle \otimes|b\rangle\}$ a general state of $\mathcal{H}$ can be written as

$$
|\psi\rangle=\sum_{f}^{D_{f}} \sum_{b}^{D_{b}} c_{f, b}|s\rangle
$$

In the following we focus on the new transport model (1) and the spinless Holstein model $(9)$, where the fermionic $\left(D_{f}=\left(\begin{array}{c}N \\ N_{f}\end{array}\right)\right)$ and bosonic $\left(D_{b}=\right.$ $\left.\left(\begin{array}{c}N+N_{b} \\ N_{b}\end{array}\right)\right)$ basis states can be defined as follows:

$$
|f\rangle=\prod_{i=1}^{N}\left(c_{i}^{\dagger}\right)^{n_{i, f}}|0\rangle_{f} \quad \text { and } \quad|b\rangle=\prod_{i=1}^{N} \frac{1}{\sqrt{m_{i, b} !}}\left(b_{i}^{\dagger}\right)^{m_{i, b}}|0\rangle_{b} .
$$

Here $n_{i, f} \in\{0,1\}, m_{i, b} \in\left\{0, \ldots, N_{b}\right\}$ with $\sum_{i}^{N} m_{i, b} \leq N_{b}$ holds, and $N_{f}=$ $\sum_{i}^{N} n_{i, f}$ is the number of fermions. Parallelisation of the MVM step can easily be done by distributing the fermionic elements to the parallel processes, i.e. the coefficients of a general state are mapped to the processes $\mathbf{P}_{i d}(0 \leq i d \leq$ $n p-1$ ) as follows (assuming the number of processes $(n p)$ being a divisor of $\left.D_{f}\right)$ :

$$
\left\{c_{f, b} ; f=i d * \frac{D_{f}}{n p}+1, \ldots,(i d+1) * \frac{D_{f}}{n p} ; b=1, \ldots, D_{b}\right\} \stackrel{\mathbf{F}}{\rightarrow} \mathbf{P}_{i d} .
$$

If one is free to choose $n p$ as a divisor of $D_{f}$ this distribution makes best use of the available memory. For the MVM step

$$
\left|\psi^{\text {new }}\right\rangle=\left|\psi^{\text {new }}\right\rangle+H\left|\psi^{\text {old }}\right\rangle
$$




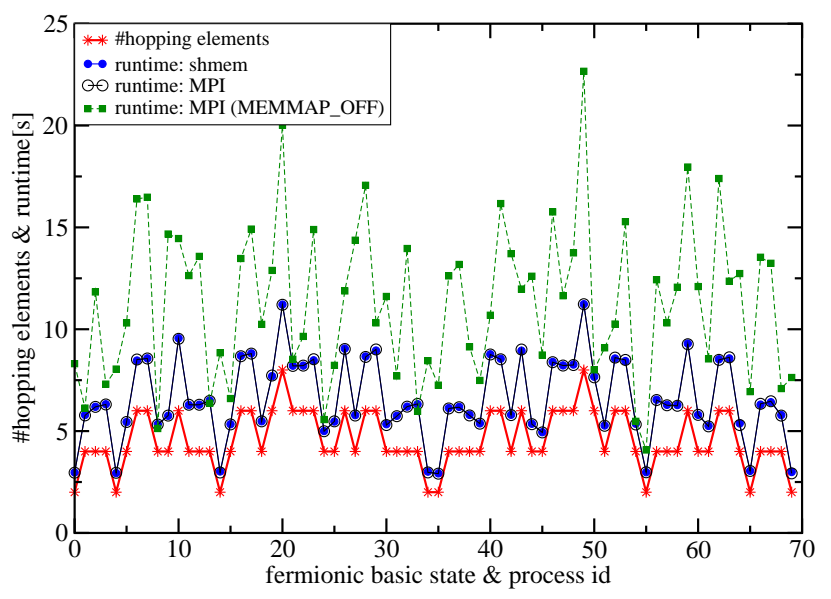

Fig. 7 The number of hopping elements of each fermionic basis state and the corresponding time required to do communication and computation for each element on 70 cores of one node of HLRB-II. The computations have been done for the Hamiltonian (1) with $N=8$ and $N_{f}=4$ (i.e. $D_{f} / n p=1$ ) as well as $D_{b}=5 \times 10^{7}$ using different implementations of the sparse MVM step.

each processor $\mathbf{P}_{i d}$ computes the update of its contribution to the left hand side vector $\left(\left\{c_{f, b}^{n e w} ; f=i d * \frac{D_{f}}{n p}+1, \ldots,(i d+1) * \frac{D_{f}}{n p} ; b=1, \ldots, D_{b}\right\}\right)$. Bosonic operators do not change the fermionic part of the basis state and thus their contribution to $H$ can be computed locally. Interprocess communication is solely generated by the fermionic operators, e.g. to compute the hopping matrix element $\left\langle\bar{f}_{1}\left|c_{i+1}^{\dagger} c_{i}\right| \bar{f}_{2}\right\rangle$ for a given $i$ the process holding $\left(\left\{c_{\bar{f}_{1}, b}^{\text {new }} ; \quad b=1, \ldots, D_{b}\right\}\right)$ needs to access the appropriate data $\left(\left\{c_{\bar{f}_{2}, b}^{\text {old }} ; \quad b=1, \ldots, D_{b}\right\}\right)$ of the r.h.s vector in Eq. (18), potentially located on a different process. The data exchange can be realized in a portable message passing interface (MPI) implementation with \{MPI_ISEND, MPI_RECV pairs or with the SGI specific shmem library through the one sided shmem_get operation. The advantages of long messages $\left(D_{b} \approx 10^{6}-10^{8}\right)$ and static communication patterns, however, are overcompensated by severe load balancing problems if $D_{f} / n p \approx 1$, which is true for most massively parallel runs. In the limits of $D_{f} / n p=1, N_{f} / N=0.5$ and a $1 \mathrm{D}$ chain with periodic boundary conditions the problem can easily be understood when the two extreme workload cases arising from the electron hopping processes are identified. Those $N$ processes holding electronic states where all (spinless) electrons are located at neighbouring sites have to fetch only two arrays of the length $D_{b}$ from other processes. Quite contrary, for the two states where the electrons occupy even and odd sites, respectively, $2 \times N_{f}$ hopping processes occur causing the same number of communication and computation steps on those processes. In Fig. 7 the load imbalance effect is presented for an 8-site system. The difference in 
runtime between the processes with highest $(11.3 \mathrm{sec})$ and lowest workload $(2.9 \mathrm{sec})$ is close to the factor of $N_{f}=4$ as given by the simple approximation above. Of course, these imbalances have substantial impact on scalability at large processor numbers, e.g. for the half-filled 16-site model $\left(D_{f}=12870\right)$ the time per MVM step increases from $40 \mathrm{sec}$ with $N_{b}=9\left(D_{t o t}=2.6 \times 10^{10}\right)$ on 300 processor cores to approximately $1800 \mathrm{sec}$ on 3328 cores when adding three more bosons $\left(D_{t o t}=3.9 \times 10^{11}\right)$ to the system. Although this is for sure one of the largest sparse matrix ED problems solved at the time of writing the additional insight gained by adding three phonons does not justify to run production jobs at that scale and performance.

Furthermore, Fig. 7 demonstrates a peculiarity of the SGI Altix system, namely single sided copies as realized in user applications by shmem_get operations. Obviously those operations are used in the MPI implementation as well through automatically replacing the $\{$ MPI_ISEND, MPI_RECV $\}$ pair by a shmem_get operation, giving the same runtime for both versions. The benefit of single sided communication for the ED application becomes clearly visible if one deactivates it, e.g. by setting the environment variable MPI_MEMMAP_OFF.

The problem described above is closely connected with the choice of the basis states and their distribution to the processors. Most strategies to alleviate the workload imbalances, e.g. distributing equal tasks to each process, would come at the costs of excessive memory overhead which usually is not acceptable.

For the Holstein model, however, the introduction of a re-mapping step for the data distribution within the MVM can balance the workload and communication but comes at the cost of massive global communication. The basic difference to the Hamiltonian (1) is that there is only unrestricted hopping of the fermions in the Holstein model, i.e. the bosonic contribution to the basis states is not affected by the fermionic hopping process, and the bosonic fluctuations couple to the local fermion density, which is diagonal in the fermionic basis states. While the latter term can be computed without communication using the mapping described above by Eq. (17) and is perfectly balanced for the spinless fermion case, the fermionic hopping contribution can be evaluated locally on each processor if the mapping between the coefficients of a general state and the processes $\mathbf{P}_{i d}$ is as follows (for the sake of simplicity $n p$ is again assumed to be a divisor of $D_{b}$ ):

$$
\left\{c_{f, b} ; f=1, \ldots, D_{f} ; b=i d * \frac{D_{b}}{n p}+1, \ldots,(i d+1) * \frac{D_{b}}{n p}\right\} \stackrel{\mathbf{B}}{\rightarrow} \mathbf{P}_{i d} .
$$

In this formulation, the bosonic dimension is distributed to the processes, i.e. for a given bosonic basis state all fermionic states reside on the same processor, allowing to compute the fermionic hopping process locally and achieving a good load balance as well.

The re-mapping step can be integrated into the MVM by introducing two MPI_Alltoall (MPI_A2A) calls allowing to switch between the two mappings 
backward and forward. Note that the actual implementation is more involved but a detailed discussion is beyond the scope of this report. The new A2A implementation can improve the time per MVM step for a typical problem configuration with $D_{f} / n p=1$ by a factor of 3.5 to 5.5 as presented in Tab. 1 . For this test case the performance gain is in the range of the reduction of the

\begin{tabular}{c|c|c} 
MVM version & arbitrary distribution & dedicated nodes \\
\hline Original & 110 & 69 \\
A2A & 20 & 20 \\
A2A-shmem & 11 & 10 \\
A2A-shmem-BW & 10 & 9
\end{tabular}

Table 1 Time per MVM step in seconds for Holstein model with $N=16, N_{f}=4$ $\left(D_{f}=1820\right), N_{b}=12\left(D_{b}=3.0 \times 10^{7}\right)$ running on 1820 cores of HLRB-II. Measurements have been done for an arbitrary distribution of the MPI processes to the 13 HLRB-II "highbandwidth" nodes (second column) and for explicitly allocating four "high-bandwidth" nodes and running 455 MPI processes on each node (third column). Please confer [33] for the actual node configuration of HLRB-II.

maximum communication traffic which is $2 \times N_{f} \times D_{b}$ words for the original implementation and approximately $2 \times D_{b}$ words for the A2A version (in principle each process has to send a message of length $D_{b} / n p$ words to all other $(n p-1)$ processes in both $\mathrm{A} 2 \mathrm{~A}$ steps). In this context one word is equivalent to eight Bytes and it is sufficient in our discussion to evaluate the outgoing data traffic only which is the same in volume as the incoming traffic.

Further improvements can be gained by replacing the MPI_A2A call by a loop over SGI specific shmem_get calls with access patterns appropriately shifted between the different processes ("A2A-shmem" version) to avoid concurrent access of several processes to the same (remote) memory. This yields an additional runtime reduction by a factor of two because there is no need to synchronise the processes after the A2A step. When a process has fetched its data it can proceed with computation while other processes can do their data transfer, allowing for overlapping of communication and computation on different processes. This effect can better be exploited by changing the program flow manually to enhance the concurrent use of processors and interconnect. In this final version ("A2A-shmem-BW") processes with even process id traverse the MVM in the standard way (left column of Fig. 8), i.e. fetch their data for the first re-mapping step, while odd process ids start with the computation of the bosonic part. Since the contribution of the bosonic part to the total runtime is only of the order of $1 \mathrm{sec}-2 \mathrm{sec}$ in the test case the effect is not as impressive as before but still matches the expectations. A further potential optimisation in this direction could be to include parts of the vector-vector operations of the outer numerical approach (e.g. Lanczos iteration) into the MVM to have more computations available for overlapping with communication. 


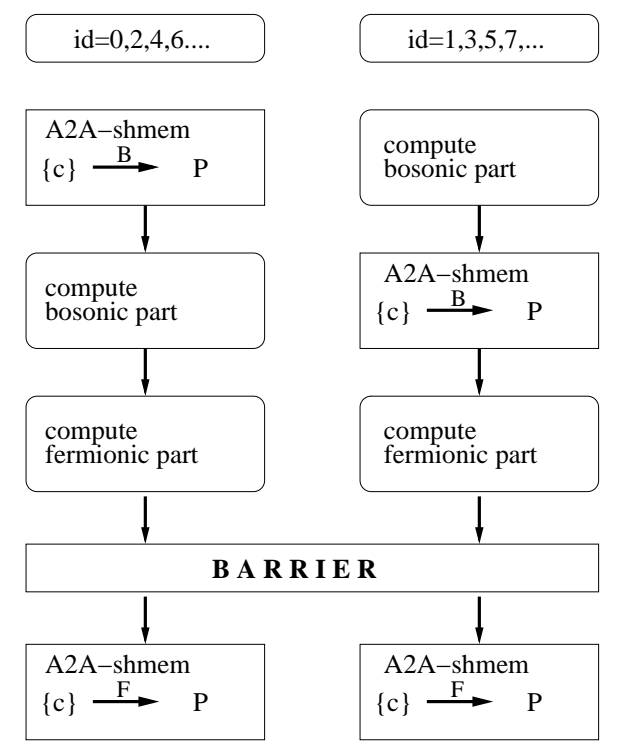

Fig. 8 Program flow in MVM for the "A2A-shmem-BW" implementation. A synchronisation of even (left) and odd processes (odd) is required before the second A2A call to ensure data consistency.

In a final step the A2A-shmem-BW variant is compared with the original implementation for a weak scaling scenario, i.e. the problem size (in this context $D_{f}$ ) is increased with increasing processor number. Tab. 2 clearly demonstrate the benefit of the A2A-shmem-BW version. It must be empha-

\begin{tabular}{c|c|c|c|c} 
processes $(n p)$ & $D_{f} / n p\left(N_{f}\right)$ & $D_{t o t}$ & original & A2A-shmem-BW \\
\hline 120 & $1(2)$ & $3.6 \times 10^{9}$ & 6.5 & 2.2 \\
560 & $1(3)$ & $17.0 \times 10^{9}$ & 34.2 & 4.1 \\
1820 & $1(4)$ & $55.3 \times 10^{9}$ & 69.3 & 9.3 \\
4368 & $1(5)$ & $132.8 \times 10^{9}$ & 158.3 & 13.7 \\
4004 & $2(6)$ & $243.4 \times 10^{9}$ & - & 28.4 \\
5720 & $2(7)$ & $347.7 \times 10^{9}$ & - & 38.2
\end{tabular}

Table 2 Time per MVM step in seconds of the original version (fourth column) and the A2A-shmem-BW implementation (fifth column) for a weak scaling scenario. The Holstein model with $N=16, N_{f}=1, \ldots, 7, N_{b}=12\left(D_{b}=3.0 \times 10^{7}\right)$ has been tested up to 5720 cores of HLRB-II. Measurements have been done for an arbitrary distribution of the MPI processes to the 13 HLRB-II "high-bandwidth" nodes. For the two largest data sets no measurements have been done with the original version due to excessive runtimes.

sised that even for weak scaling a constant time per MVM for the A2A based implementation can not be expected, since the bi-sectional bandwidth per direction and socket pair (i.e. dual-core chip) decreases on HLRB-II from 
0.8 GByte/sec within a 510 core node to 0.1 GByte/sec for the complete system [33]. The performance of the ED application decreases roughly at the same ratio as can been seen in Tab. 2. However, the optimisations described above enable reasonable runtimes for very large scale ED studies of the Holstein model on thousands of cores on HLRB-II.

ED studies involving sparse matrices of similar sizes as those presented in Tab. 2 are very rare in literature at the time of writing. For the fermionic Hubbard model which is a related problem, but easier to implement and parallelise, a record ED study with a matrix dimension of $159 * 10^{9}$ has been reported at SC05 [34].

\section{Acknowledgements}

This work was supported by the Competence Network for Technical Scientific High Performance Computing in Bavaria (KONWIHR, project HQS@HPC) and the Deutsche Forschungsgemeinschaft (SFB 652, B5). We acknowledge helpful discussions with D. M. Edwards, G. Hager and S. A. Trugman.

\section{References}

1. F.C. Zhang, T.M. Rice, Phys. Rev. B 37, 3759 (1988)

2. E. Dagotto, Rev. Mod. Phys. 66, 763 (1994)

3. T. Holstein, Ann. Phys. (N.Y.) 8, 325 (1959)

4. W.P. Su, J.R. Schrieffer, A.J. Heeger, Phys. Rev. Lett. 42, 1698 (1979)

5. H. Fehske, M. Kinateder, G. Wellein, A.R. Bishop, Phys. Rev. B 63, 245121 (2001)

6. D. M. Newns, C.-C. Tsuei, J. Phys. Conf. Ser. 92, 012007 (2007)

7. L.G.L. Wegener, P.B. Littlewood, Phys. Rev. B 66, 224402 (2002)

8. S. Komineas, G. Kalosakas, A.R. Bishop, Phys. Rev. E 65, 061905 (2002)

9. W.F. Brinkman, T.M. Rice, Phys. Rev. B 2 (1970)

10. C.L. Kane, P.A. Read, D. Newns, Phys. Rev. B 39, 6880 (1989)

11. S.A. Trugman, Phys. Rev. B 37, 1597 (1988)

12. G.D. Mahan, Many-Particle Physics (Kluwer Academic/Plenum Publishers, New York, 2000)

13. A.L. Chudnovskiy, Europhys. Lett. 69, 609 (2005)

14. D.M. Edwards, Physica B 378-380, 133 (2006)

15. A. Alvermann, D.M. Edwards, H. Fehske, Phys. Rev. Lett. 98, 056602 (2007)

16. A. Weiße, G. Wellein, A. Alvermann, H. Fehske, Rev. Mod. Phys. 78, 275 (2006)

17. E. Jeckelmann, H. Fehske, La Rivista del Nuovo Cimento 30, 259 (2007)

18. A. Weiße, H. Fehske, in Computational Many-Particle Physics, ed. by H. Fehske, R. Schneider, A. Weiße, Lect. Notes Phys. 739 (Springer, Berlin Heidelberg, 2008), pp. $529-577$

19. G. Wellein, H. Fehske, Phys. Rev. B 58, 6208 (1998)

20. H. Fehske, S.A. Trugman, in Polarons in Advanced Materials, ed. by A.S. Alexandrov (Springer, Dordrecht, 2007), pp. 393-461

21. J.R. Schrieffer, X.G. Wen, S.C. Zhang, Phys. Rev. Lett. 60, 944 (1988) 
22. G. Grüner (ed.), Density waves in solids (Perseus Publishing, Cambridge, Massachusetts, 1994)

23. R. Peierls, Quantum theory of solids (Oxford University Press, Oxford, 1955)

24. H. Fehske, G. Wellein, G. Hager, A. Weiße, A.R. Bishop, Phys. Rev. B 69, 165115 (2004)

25. M. Hohenadler, G. Wellein, A.R. Bishop, A. Alvermann, H. Fehske, Phys. Rev. B 73, 245120 (2006)

26. H. Fehske, E. Jeckelmann, in Polarons in Bulk Materials and Systems With Reduced Dimensionality, International School of Physics Enrico Fermi, vol. 161, ed. by G. Iadonisi, J. Ranninger, G. De Filippis (IOS Press, Amsterdam, 2006), International School of Physics Enrico Fermi, vol. 161, pp. 297-311

27. G. Wellein, H. Fehske, A. Alvermann, D.M. Edwards, Phys. Rev. Lett. 101, 136402 (2008)

28. G. Wellein, H. Röder, H. Fehske, Phys. Rev. B 53, 9666 (1996)

29. J. Bonča, S.A. Trugman, I. Batistić, Phys. Rev. B 60, 1633 (1999)

30. J.K. Cullum, R.A. Willoughby, Lanczos Algorithms for Large Symmetric Eigenvalue Computations, Progress in scientific computing, vol. I \& II (Birkhäuser, Boston, 1985)

31. E.R. Davidson, J. of Comp. Phys. 17, 87 (1975)

32. R.N. Silver, H. Röder, Phys. Rev. E 56, 4822 (1997)

33. http://www.Irz-muenchen.de/services/compute/hlrb/batch/batch.html

34. S. Yamada, T. Imamura, M. Machida, in SC2005 Conference on High Performance Networking and Computing (IEEE Computer Society, 2005), p. 44 